\title{
ON A THEOREM OF LACHLAN AND MARTIN
}

GERALD E. SACKS ${ }^{1}$

In [3] we raised the following question: does there exist a recursively enumerable degree $d$ such that $O^{(n)}<d^{(n)}<O^{(n+1)}$ for all $n \geqq 0$ ? This question was answered affirmatively by Lachlan [1] and by Martin [2]. Lachlan's proof combines a familiar priority argument with the fixed point theorem of Kleene. Martin's proof is a new form of priority argument based on Theorem 3 of section 6 of [3]. In this paper we give a vanishingly short proof of the Lachlan-Martin result without any use of priority. Our argument is an exercise in the fixed point theorem. We exploit, possibly for the first time, a uniformity concealed in most proofs of the fixed point theorem.

Let $A$ be an arbitrary set of natural numbers, and let $W_{0}^{A}, W_{1}^{A}$, $W_{2}^{A}, \cdots$ be a standard simultaneous enumeration of all sets recursively enumerable in $A$. For each $e \geqq 0$, let

$$
R_{e}^{A}=\{2 n \mid n \in A\} \cup\left\{2 n+1 \mid n \in W_{\bullet}^{A}\right\} .
$$

Then $R_{0}^{A}, R_{1}^{A}, R_{2}^{A}, \cdots$ is a standard simultaneous enumeration of sets recursively enumerable in $A$ and of degree greater than or equal to that of $A$.

The proof of Theorem 3 of $\S 6$ of [3] makes clear that there exists a recursive function $t$ such that

$$
(A)(e)\left[\boldsymbol{A}<\boldsymbol{R}_{t(e)}^{A}<A^{\prime} \text { and } \quad\left(\boldsymbol{R}_{t(e)}^{A}\right)^{\prime}=\boldsymbol{R}_{\bullet}^{A^{\prime}}\right. \text { l, }
$$

where $\boldsymbol{A}$ is the degree of $A$. By the fixed point theorem, there exists a $c$ such that

$$
\text { (A) }\left[R_{t(c)}^{A}=R_{c}^{A}\right] \text {. }
$$

The fact that $c$ is independent of $A$ is the key that unlocks the shiny little box containing our proof. It follows that

$$
(A)\left[A<R_{c}^{A}<A^{\prime} \text { and }\left(R_{c}^{A}\right)^{\prime}=R_{c}^{A^{\prime}}\right]
$$

But then

$$
(A)\left[A<R_{c}^{A}<A^{\prime}<\left(R_{c}^{A}\right)^{\prime}<A^{\prime \prime}\right]
$$

and

Received by the editors December 27, 1965.

1 The preparation of this paper was supported by U. S. Army Contract ARO-D. 373. 


$$
(A)(n)\left[A^{(n)}<\left(R_{c}^{A}\right)^{(n)}<A^{(n+1)}\right] .
$$

Let $J$ and $C$ be operators such that $J(A)=A^{\prime}$ and $C(A)=R_{c}^{A}$. Then our argument (as well as that of Martin [2]) shows that $J C=C J$. In other words we have found a solution of Post's problem which commutes with the jump operator up to degrees. A. Nerode pointed out that the version of the fixed point theorem used above is better formulated as a fixed point theorem for a class of effective transformations acting on a class of effective operators. From his point of view, it is not surprising that one should use a fixed point argument to study the equation $J X=X J$.

We leave the reader with the following open question: does there exist a solution to Post's problem well defined on degrees? More precisely, does there exist a Gödel number $c$ such that for all $A$ and $B$, if $A=B$, then $R_{c}^{A}=R_{c}^{B}$ and $A<R_{c}^{A}<A^{\prime}$ ?

\section{REFERENCES}

1. A. H. Lachlan, On a problem of G. E. Sacks, Proc. Amer. Math. Soc. 16 (1965), 972-979.

2. D. A. Martin, On a question of G. E. Sacks, J. Symbolic Logic 31 (1966), 66-69.

3. G. E. Sacks, Degrees of unsolvability, Annals of Mathematics Studies No. 55, Princeton Univ. Press, Princeton, N. J., 1963.

Cornell University 